\title{
Malignant transformation of thymoma in recipient rats by heterotopic thymus transplantation from HTLV-I transgenic rats
}

\author{
Takahiro Tsuji ${ }^{1,2}$, Hitoshi Ikeda ${ }^{1,3}$, Takahiro Tsuchikawa ${ }^{1}$, Kazunori Kikuchi ${ }^{1,4}$, \\ Tomohisa Baba ${ }^{1}$, Akihiro Ishizu ${ }^{1}$ and Takashi Yoshiki ${ }^{1,5}$ \\ ${ }^{1}$ Department of Pathology/Pathophysiology, Division of Pathophysiological Science, Hokkaido University \\ Graduate School of Medicine, Sapporo, Japan
}

\begin{abstract}
Transgenic rats expressing the $p X$ gene of human T lymphocyte virus type-I (HTLV-I) under control of the rat lymphocyte-specific protein tyrosine kinase type-I promoter (Ick-pX rats) developed benign epithelial thymomas. When the thymuses of newborn Ick-pX rats were transplanted into the subcapsular space of the kidney in other thymectomized Ick-pX rats, similar tumors developed in the transplanted thymuses. Following the tumor growth, dissemination in the abdominal cavity and distant metastasis occurred. The tumors were histopathologically similar to the original thymomas, but prominent nuclear atypia and high mitotic activity were present. The Ki-67 index was twice as high as that in the originals. The tumors were transplantable into the subcutis of Ick-pX rats, although transplantation of the originals never succeeded. All evidence indicated that malignant transformation of thymoma was induced by the heterotopic transplantation. Expression of the $p X$ transgene in the transformed tumors were significantly reduced. Among host genes, the expression of p16ink4a/ARF, which was significantly upregulated in the originals, was never detected in the transformed tumors. Genomic Southern blots and PCR suggest that homozygous deletion of the p16ink4a/ARF gene may play important roles in malignant transformation in this model. Our model described here is a useful unique model for in vivo malignant transformation.
\end{abstract}

Laboratory Investigation (2005) 85, 851-861. doi:10.1038/labinvest.3700292; Published online 16 May 2005

Keywords: HTLV-I; transgenic rat; epithelial thymoma; malignant transformation; p16ink4a/ARF

Human T lymphocyte virus type-I (HTLV-I) is the etiologic agent of adult T-cell leukemia/lymphoma (ATL/L). ${ }^{1-3}$ Tax protein encoded by the unique $p X$ region of HTLV-I genome is thought to play central roles in the tumorigenesis, since it could induce immortalization of human $\mathrm{T}$ cells ${ }^{4}$ and transform fibroblast cell lines in vitro, ${ }^{5}$ and transgenic animals carrying the $p X$ gene developed several kinds of tumors in vivo. ${ }^{6-10}$ Tax activates not only transcrip-

Correspondence: Dr A Ishizu, MD, PhD, Department of Pathology/ Pathophysiology, Division of Pathophysiological Science, Hokkaido University Graduate School of Medicine, Kita-15, Nishi-7, Kita-ku, Sapporo 060-8638, Japan.

E-mail: aishizu@med.hokudai.ac.jp

${ }^{2}$ Current address: Department of Medical Microbiology, University College Dublin, Belfield, Dublin 4, Ireland.

${ }^{3}$ Current address: Section of Pathology, Hakodate Central General Hospital, Hakodate 040-8585, Japan.

${ }^{4}$ Current address: Department of Pathology, Tsukuba Medical Center Hospital, Tsukuba 305-8558, Japan.

${ }^{5}$ Current address: Genetic Lab Co. Ltd, Sapporo 060-0009, Japan.

Received 17 November 2004; revised 27 March 2005; accepted 29 March 2005; published online 16 May 2005 tion of the viral genes but also many cellular genes, such as lymphokines, interleukin-2 receptors, $C$-fos, and $c$-jun etc., through modulation of host transcription factors, such as cAMP responsive element binding factor (CREB), nuclear factor (NF)- $\kappa \mathrm{B}$ and serum responsive factor (SRF). ${ }^{11}$ In addition, Tax inhibits functions of several cell cycle regulating proteins, such as p53, tumor suppresser protein; ${ }^{12,13}$ p16ink4a, inhibitor of cyclin-dependent kinases (Cdk); ${ }^{14}$ and hsMAD1, spindle checkpoint protein. ${ }^{15}$ Tax exerts its tumorigenicity through these pleiotropic effects on host cellular genes. ${ }^{16,17}$

To investigate tumorigenicity of the $p X$ gene in lymphoid tissues, we established a transgenic rat model carrying the $p X$ gene under control of the rat lymphocyte-specific protein tyrosine kinase (p56lck) proximal promoter. ${ }^{10}$ In the transgenic rat model, epithelial thymomas frequently developed. Although fatal dyspnea sometimes occurred due to expansive growth of the thymomas in the thoracic cavity, the tumors never invaded to neighboring organs or metastasized, indicating a benign behavior. 
In the present study, we induced in vivo malignant transformation of the epithelial thymomas by heterotopic transplantation of the thymuses from newborn lck-pX rats into the subcapusular space of kidneys of other lck-pX rats which had been undergone thymectomy, and compared the biological and molecular characteristics between the original and transformed tumors to better understand the roles of the $p X$ gene in tumorigenesis in vivo.

\section{Materials and methods}

\section{Rats}

Inbred male Fisher 344/jcl rats (F344 rats) were purchased from Clea Japan (Osaka, Japan). The Tg38 line of lck-pX rats, which established in F344 strain and frequently had thymomas, ${ }^{10}$ was maintained at the Institute for Animal Experimentation, Hokkaido University Graduate School of Medicine, and was used in this study. All animal experiments were performed according to the Guide for the Care and Use of Laboratory Animals in Hokkaido University Graduate School of Medicine (http://www.hokudai. ac.jp/animal/houki/hokudaisisin.html).

\section{Histopathology and Immunohistochemistry}

Tissues from lck-pX and nontransgenic F344 rats were fixed in $10 \%$ phosphate-buffered formaldehyde and embedded in paraffin blocks. Each $4 \mu \mathrm{m}$ section was stained with hematoxylin and eosin (HE). For Ki-67 immunostaining, sections were autoclaved for $10 \mathrm{~min}$ at $120^{\circ} \mathrm{C}$ and then reacted with mouse anti-rat Ki-67 monoclonal antibody (DAKO, Glostrup, Denmark). Specific staining was detected using an LSAB2 kit (DAKO). Nuclei with positive staining for Ki-67 were counted in 10 highpower $(\times 400)$ fields of each sample. Any nuclear staining, regardless of its intensity, was considered to be positive. Ki-67 staining index was expressed as percentage of the positive nuclei.

\section{Transplantation of Thymus into the Subcapsular Space of the Kidney}

Thymuses were extirpated from newborn lck-pX rats and then chopped into small fragments. The fragments were transplanted into the subcapsular space of bilateral kidneys of lck-pX rats (6 weeks old) at 1 week after thymectomy. Right and left lobes of the thymus from one donor were transplanted into bilateral kidneys, respectively, of one recipient. Totally, 5 times of independent transplantation were performed.

\section{Tumor Transplantation into the Subcutis of lck-pX Rats}

About $25 \mathrm{~mm}^{3}$ of tumor tissues were cut into small fragments in $5 \mathrm{ml}$ Hanks' balanced salt solution
(HBSS) (Invitrogen, Co., Carlsbad, CA, USA). The fragments in $0.5 \mathrm{ml}$ HBSS were injected into the subcutis of bilateral back of lck-pX rats (7 weeks old). The tumor from one donor was transplanted into one recipient rat. Totally, 3 times of independent transplantation were performed. At 3 months after the injection, tumor formation was macroscopically and microscopically evaluated. Recipient rats used in this experiment had not been given thymectomy. Since it was considered that Tax (protein product of the $p X$ transgene) would be recognized by immune systems in nontransgenic recipients, lck-pX rats served as recipients in our transplantation experiments.

\section{Nucleic Acid Isolation}

Tumors and thymuses from lck-pX and nontransgenic F344 rats were snap-frozen in liquid nitrogen prior to isolation of RNA and DNA. Before being frozen, thymocytes were disaggregated by rinsing the thymuses, the surface of which had been cut, in HBSS. It was histologically confirmed that over $90 \%$ of thymocytes were removed. Total RNAs were isolated using ISOGEN reagents (Nippon Gene, Toyama, Japan) and genomic DNAs were isolated by the standard phenol-chloroform extraction. All samples were microscopically confirmed to be the target tissues.

\section{Real-Time Quantitative Reverse Transcriptase-Polymerase Chain Reaction}

Complementary DNAs were synthesized from total RNAs using $2 \mu \mathrm{g}$ of DNaseI (TAKARA Shuzo, Co., Kyoto, Japan), a random primer set (TAKARA Shuzo, Co.) and Moloney murine leukemia virus RT (Invitrogen, Co.). Real-time quantitative reverse transcriptase-polymerase chain reaction (RT-PCR) was performed using the ABI PRISM 7900 Sequence Detection System (Applied Biosystem, Foster City, CA, USA) with SYBR Green I as a double strand DNA-specific binding dye, and the reaction was continuously monitored based on the fluorescence levels, according to Wittwer et al. ${ }^{18}$ Each cDNA was amplified using QuantiTect SYBR Green Master Mix (QIAGEN, Hilden, Germany) containing $0.3 \mu \mathrm{M}$ of the specific primers for each gene in a total volume of $10 \mu \mathrm{l}$, according to the following PCR conditions: $95^{\circ} \mathrm{C}$ for $15 \mathrm{~min}$, followed by 45 cycles of $94^{\circ} \mathrm{C}$ for $30 \mathrm{~s}$ and $60^{\circ} \mathrm{C}$ for $30 \mathrm{~s}$ and $72^{\circ} \mathrm{C}$ for $1 \mathrm{~min}$. Melting curve analysis was performed at the end of each reaction, after which absence of primer dimmers was confirmed. Standard curves for each gene were generated from a serially diluted cDNA mixture that consisted of cDNAs from five discrete samples. Glyceraldehyde-3-phosphate dehydrogenase (GAPDH) was used as a control for the amount of RNAs. Each reaction was performed in triplicate to calculate the 
average and standard deviation. Primer sets used are listed in Table 1.

\section{Southern Blotting}

In all, $20 \mu \mathrm{g}$ of genomic DNAs from each sample digested either with HindIII, BamHI or EcoRI was electrophoretically separated on a $0.8 \%$ agarose gel and transferred to a Hybond $\mathrm{N}^{+}$membrane (Amersham Pharmacia Biotech., Buckinghamshire, UK) using the standard capillary method. The digoxygenin (DIG)-labeled cDNA probes were prepared by PCR amplification. Briefly, $1 \mu \mathrm{g}$ of cDNAs from a normal rat thymus was amplified in a total of $50 \mu \mathrm{l}$ solution containing $1 \mu \mathrm{g}$ cDNA template, $70 \mu \mathrm{M}$ DIG11-dUTP (alkali-label), $\quad 130 \mu \mathrm{M} \quad$ dTTP, $200 \mu \mathrm{M}$ dATP:dGTP:dCTP, $5 \mathrm{mM} \mathrm{MgCl}_{2}, 5 \mu \mathrm{l} 10 \times \quad$ PCR buffer II, 3.75 U AmpriTaq Gold (Roche Diagnostics, Mannheim, Germany), and the primer set for the rat p16ink4a gene, sense (5'-CATCTCCGAGAGGAAG GCG-3') and antisense (5'-TCTCGCGTTGCCAGAA GTG-3'). After denaturing at $100^{\circ} \mathrm{C}$ for $5 \mathrm{~min}$, the DIG-labeled probe was hybridized to the membrane in DIG Easy Hyb (Roche Diagnostics) at $45^{\circ} \mathrm{C}$ overnight. Next, the membrane was washed twice in $2 \times$ SSC solution $(1 \times$ SCC: $0.15 \mathrm{M}$ sodium citrate, $15 \mathrm{mM}$ citric acid, $\mathrm{pH} 7.0$ ) that contained $0.1 \%$ sodium dodecyl sulfate (SDS) at room temperature for $15 \mathrm{~min}$, and then washed twice in $0.5 \times$ SSC and $0.1 \%$ SDS at $65^{\circ} \mathrm{C}$ for $15 \mathrm{~min}$. Hybridized probes were visualized by chemiluminescense using anti-DIG-
$\mathrm{F}(\mathrm{ab})$ conjugated with alkaline phosphatase (Roche Diagnostics) and CDP-star (New England Biolabs, Beverly, MA, USA) as a TIFF format image using Fluor-S MultiImager (Bio-Rad Laboratories, Hercules, CA, USA). Densities of bands with specific hybridization were calculated using Scion image software (Scion, Co., Frederick, MD, USA). After dehybridization, the membrane was rehybridized with the rat $R b$ probe prepared using the same procedure described and the primer set, sense ( $5^{\prime}$-GGAGCACAGCGATAT AAACTTGG- $3^{\prime}$ ) and antisense (5'-CTGCTTGCGT CTCTGTATTTGC- $3^{\prime}$ ), as a control.

\section{In Vitro Cell Cloning from the Transformed Tumors and Genomic PCR}

Tumors with malignant transformation, which developed in recipient rats, were cut into small fragments in HBSS, then placed onto Petri dishes and cultivated in Dulbeccos modified Eagle's medium (Invitrogen, Co.) supplemented with 10\% fetal bovine serum for 1 week. After two passages, adherent tumor cells were stripped with trypsinEDTA solution, and then cloned using the limiting dilution method ( 0.3 cells/well) in 96-well plates. In all, $200 \mathrm{ng}$ of genomic DNAs extracted from the cloned cells was used as templates for PCR with the primer sets for the p16ink4a/ARF, similar to 5-methylthioadenosine phosphorylase (MTAPase), and $R b$ genes (see Table 2). The PCR was performed in a total of $20 \mu \mathrm{l}$ solution, containing DNA template,

Table 1 Primer sets used in the real-time quantitative RT-PCR experiments

\begin{tabular}{llll}
\hline Name of genes & Sense primer $\left(5^{\prime}-3^{\prime}\right)$ & Antisense primer $\left(5^{\prime}-3^{\prime}\right)$ & Accession no. \\
\hline HTLV-I $p X$ & ATCCCGTGGAGACTCCTCAA & CCAAACACGTAGACTGGGTA & M10085 \\
$p 16$ ink $4 a$ & CCAAACGCCCCGAACAC & CACTTTGACGTTGCCCATCA & NM_031550 \\
ARF & TTCGTGGTCACTGTGAGGATTC & CCAGGAACTGCACCAAGAAAA & L81168 \\
Cdk 4 & TCGTGAGGTGGCCTTGTTAAG & GACATCCATCAGCCGTACAACAT & L11007 \\
Cyclin $D 1$ & ATGTTTGCGGATGATCTGCTT & CACGATTTCATCGAACACTTCCT & D14014 \\
Cyclin $D 2$ & ACATCGGTGTGGGTGATCTTG & AGCAGGACGAGGAAGTGATG & L09752 \\
Cyclin $D 3$ & GTGCGGCTTGATCTCCTTTT & GCTTGGAGGAGCGCTACGT & D16309 \\
Rb & CCAAGAGCAAACATCACACATGT & CTGGAGGGAGTCCTAGCAACTC & L07126 \\
$p 53$ & TCCTCTGTCCGACGGTCTCT & TTCTGGGACGGGACAGCTT & X13058 \\
$p 21$ & CATGAGCGCATCGCAATC & CCTGTTCCACACAGGAGCAA & U24174 \\
mdm2 & AATCATGGTCATAGCTGGTTTCC & ATGCTTCCGGCTTCGTATGTT & X58876 \\
GADD45 & GCTGACTCCGAGCCTTGCT & AGCAGAAGATCGAAAGGATGGA & L32591 \\
Bax & CCCACCAGCTCTGAACAGTTC & GTGTCTCCCCAGCCATCCT & NM_017059 \\
AAPDH & ATGGGAGTTGCTGTTGAAGTCA & CCGAGGGCCCACTAAAGG & M17701 \\
\hline
\end{tabular}

Table 2 Primer sets used in the genomic PCR experiments

\begin{tabular}{lll}
\hline Name of genes & Sense primer $\left(5^{\prime}-3^{\prime}\right)$ & Antisense primer $\left(5^{\prime}-3^{\prime}\right)$ \\
\hline p16ink $4 a$ & & \\
Exon $1 \beta$ & TTCGTGGTCACTGTGAGGATTC & CCAGGAACTGCACCAAGAAAA \\
Exon $1 \alpha$ & CCAAACGCCCCGAACAC & CACTTTGACGTTGCCCATCA \\
Exon 2 & GTGATGATGATGGGCAACGTC & TCGCAGTTCGAATCTGCACC \\
Exon 3 & TGGCTTCACTTCTGGCAAC & CTCCCTCCCTCTGCTAACCT \\
Similar to 5-methylthio-adenosine phosphorylase & AGGCGTGCTGTGTTTCTGTT & TTGGGGAAGAAGGTTGTTTG \\
Rb & CCAAGAGCAAACATCACACATGT & CTGGAGGGAGTCCTAGCAACTC \\
\hline
\end{tabular}


$200 \mu \mathrm{M}$ dNTPs, $5 \mathrm{mM} \mathrm{MgCl}_{2}, 2 \mu \mathrm{l}$ of $10 \times$ PCR buffer II, $1 \mathrm{U}$ of AmpriTaq Gold, and the primer set, at 35 cycles under the same thermal conditions for real-time RT-PCR. The PCR products were electrophoretically separated on a $4 \%$ agarose gel and visualized by staining with ethidium bromide.

\section{Karyotype Analysis}

Chromosomes of cells separated from the original and transformed thymomas were analyzed, according to Wang and Fedoroff. ${ }^{19}$ A total of 17 and 18 cells from both original and transformed thymomas were analyzed, respectively.

\section{Results}

\section{Development of Thymomas with Malignant Phenotypes in Heterotopically Transplanted lck-pX Thymuses in the Subcapusular Space of Recipient Rat Kidney}

At 3 months after the thymus transplantation, tumors developed in the transplanted thymuses in the subcapusular space of the recipient lck-pX rat kidney (Figure 1a). Histologically, tumors were composed predominantly of spindle-shaped cells, and these cells showed medullary growth with storiform and whirly patterns (Figure 1b). Immunohistochemically, tumor cells were positive for cytokeratin (data not shown). These were the same histopathological features as original thymomas in lck-pX rats. ${ }^{10}$ Abdominal distention in recipient rats became evident from 6 months after the transplantation. In these rats, large tumors with ascites were found in contact with the kidney, and peritoneal disseminations and distant metastases to the lungs and liver were evident (Figure 1c, d). These phenotypes indicated that the tumors were highly malignant. This phenomenon was observed in all cases where heterotopic transplantation had been performed $(n=5)$.

Microscopically, the tumor cells had larger nuclei and showed more prominent nuclear atypia compared to the original thymomas (Figure 1e, f). Distinct nucleoli were evident and mitotic activity was high (Figure 1e). The Ki-67 immunoreactivity, which assesses the proliferative activity of tumor cells, was significantly higher and stronger than that seen in the original thymomas (Figure 1g, h). Mean values and standard deviations of Ki-67 staining indices were $55.8 \pm 7.1 \%$ in thymomas with malignant transformation $(n=5)$ and $25.9 \pm 4.3 \%$ in original thymomas $(n=9)$, with a statistical significance $(P<0.001)$ (Figure 1i).

To assess the biological malignancy, tumor tissue fragments were injected subcutaneously into lck-pX rats. In all rats injected with the transformed thymoma tissues $(n=3)$, tumors in the subcutis rapidly grew within 1 month (Figure 2a). On the other hand, tumor formation never observed in any rat injected with the original thymoma tissues $(n=6)$ (Figure $2 b)$ until 3 months after the injection, both macroscopically and microscopically (data not shown).

\section{Gene Expression Levels in the Original and Transformed Thymomas, Using Real-Time Quantitative RT-PCR}

To compare the transcription levels of genes between the original and transformed thymomas, real-time quantitative RT-PCR was performed. Since abnormalities of p53 and Rb pathways were reported to play important roles in tumorigenesis of many human malignancies including ATL ${ }^{20}$ we mainly quantified the expression levels of genes associated with these pathways. In Figure 3, HTLV-I $p X$, which is a transgene, was detected at high levels in the original thymomas, but at low levels in the transformed thymomas similar to what were seen in the tumor-free thymuses of lck-pX rats. No expression of p16ink4a and $A R F$ was evident in the thymomas with malignant transformation, although these expressions were consistently high in the originals. Expression of cyclin D2 showed a pattern similar to that seen in HTLV-I $p X$. These alterations in gene expression levels in the transformed thymomas were significant. Although no significant difference was evident, expression levels of $C d k 4$ and $m d m 2$ tended to be low in the thymomas with malignant transformation compared with those of the originals, and cyclin D3, GADD45 and $R b$ genes in both types of thymomas were expressed at low levels compared with those of the tumor-free thymuses of lck-pX rats.

\section{Genomic Analysis of the p16/ARF Locus in the Transformed Thymomas}

To determine why the expressions of p16ink4a and $A R F$ disappeared with malignant transformation, we analyzed the genome of cells with malignant transformation. Normal rat karyotype 42XY was seen in the original thymomas, ${ }^{21}$ but several alterations in karyotype were evident in the cells with malignant transformation (eg 41XY, -10). However, no homozygous alteration in karyotype was evident on chromosome 5 that codes the p16ink4a/ARF locus in rats. For a further assessment, genomic Southern blot analysis with three different restriction enzymes (HindIII, BamHI and EcoRI) was performed using the p16ink $4 a$ cDNA probes. Regardless of the restriction enzyme, there was no difference in banding patterns among the transformed and original thymomas and the thymus of a nontransgenic rat (Figure 4a). However, densities of all bands in the transformed thymomas were significantly low compared with those of the 
original thymomas and the nontransgenic thymus. Each density of the HindIII digested band in genomes of three transformed thymomas tested was low (10.3, 22.7 and 19\%, respectively) compared with that of the normal rat genome when the density was calculated based on that of the
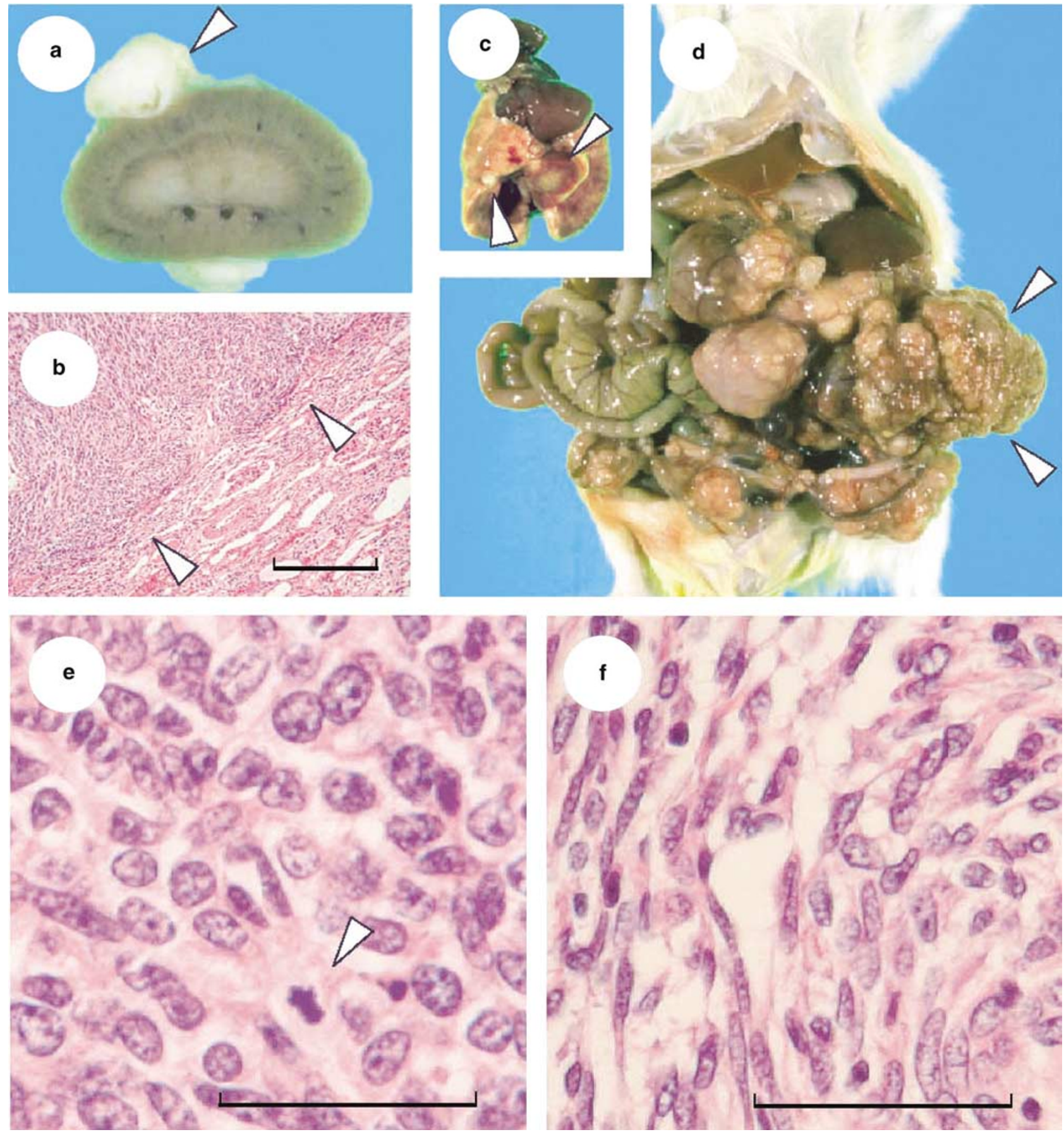

Figure 1 Development of thymomas in the lck-pX thymuses transplanted into the subcapusular space of the recipient kidney, and the malignant transformation. (a) At 3 months after the transplantation, a tumor (arrowhead) developed in the transplanted lck-pX thymus. (b) Microscopically, the tumor (arrowheads) showed the same histological features as original thymomas in lck-pX rats. (c) Metastases to the lungs (arrowheads). (d) Peritoneal disseminations and a large tumor formation adjacent to the kidney (arrowheads) were observed when the tumor was incubated for another 3 months. Figures are from representative rats at 3 and 6 months after the transplantation $(n=5)$. (e, f) Histopathologically, nuclear atypia and mitosis (e, arrowhead) were prominent in the transformed thymomas compared with those in the originals (f) (HE staining). (g, h) Many nuclei of cells positively stained with anti-Ki-67 antibody were found in the transformed thymomas (g, brown staining in nuclei) but few in the originals (h). Bars in microscopic photos indicate $250 \mu \mathrm{m}$ (b), and $50 \mu \mathrm{m}(\mathbf{e}-\mathbf{h})$. (i) Comparison of Ki-67 index between the transformed ( $n=5$, closed circles) and original ( $n=9$, open circles) thymomas. Bars indicate mean \pm standard deviation. *Student's $t$-test was used for statistical analysis. 

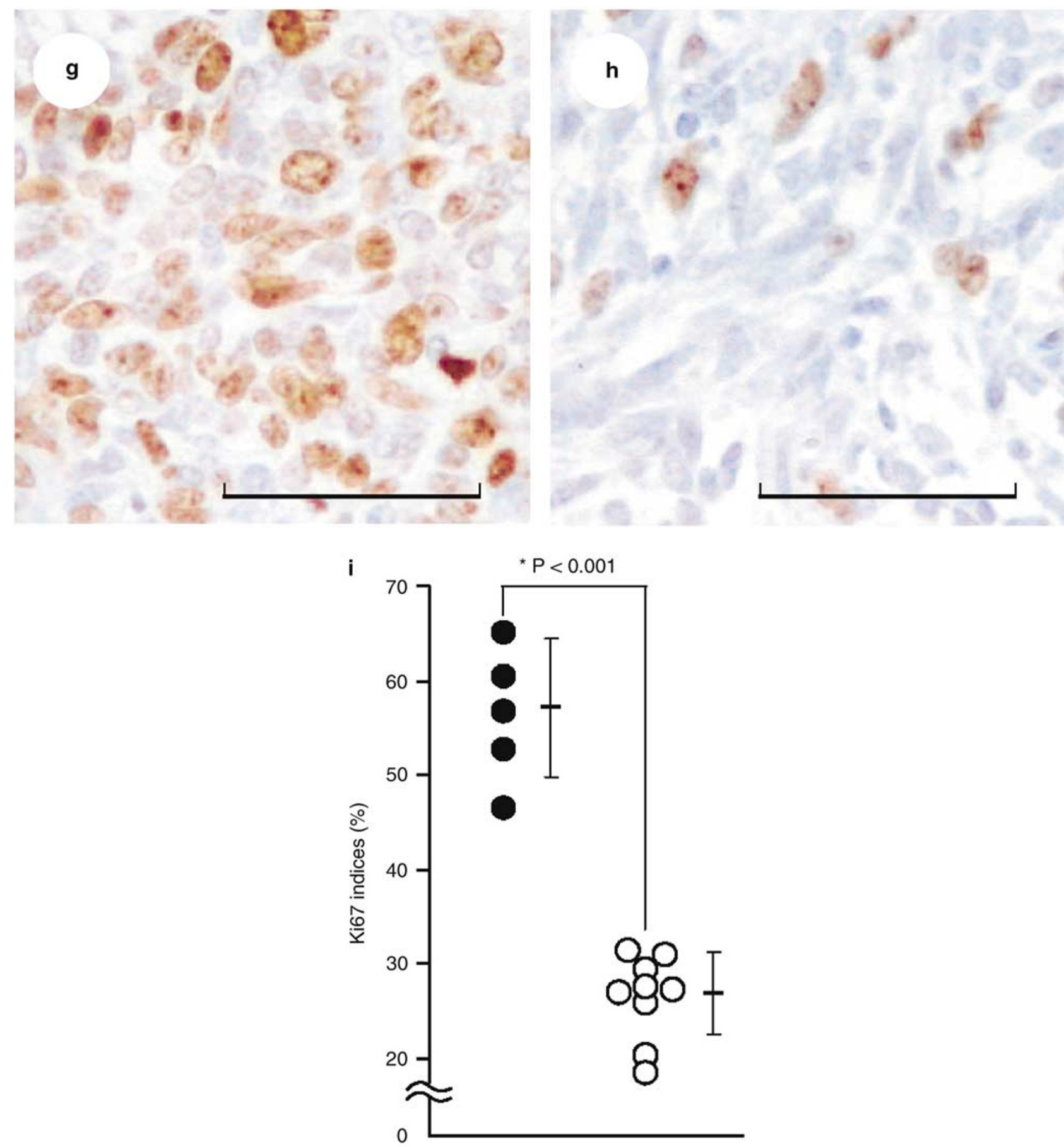

Figure 1 Continued.

nonaffected $R b$ gene in each sample (Figure 4b). Since samples contained nontumor tissues, such as vascular and other mesenchymal components, it was suggested that the p16ink4a/ARF locus might be homozygously deleted in the thymomas with malignant transformation. No significant difference in the density of p16ink4a bands was found between the original thymomas and the nontransgenic thymus. To confirm homozygous deletion of the p16ink4a/ARF locus in the transformed thymomas, we established cell clones from the disseminated tumor (used in Figure 4b, lane 7) and analyzed by genomic PCR using the primer set for the p16ink $4 a$ exon $1 \alpha$. No band corresponded to the p16ink $4 a$ exon $1 \alpha$ was amplified in all three cell clones tested, although the band corresponded to the $R b$ gene was evident at the same levels as that seen in a control rat genomic DNA (Figure 5). Rat p16ink4a/ARF gene is composed of exons $1 \beta, 1 \alpha, 2$ and 3 , followed by the gene similar to 5-MTAPase (Figure 6a). When we 

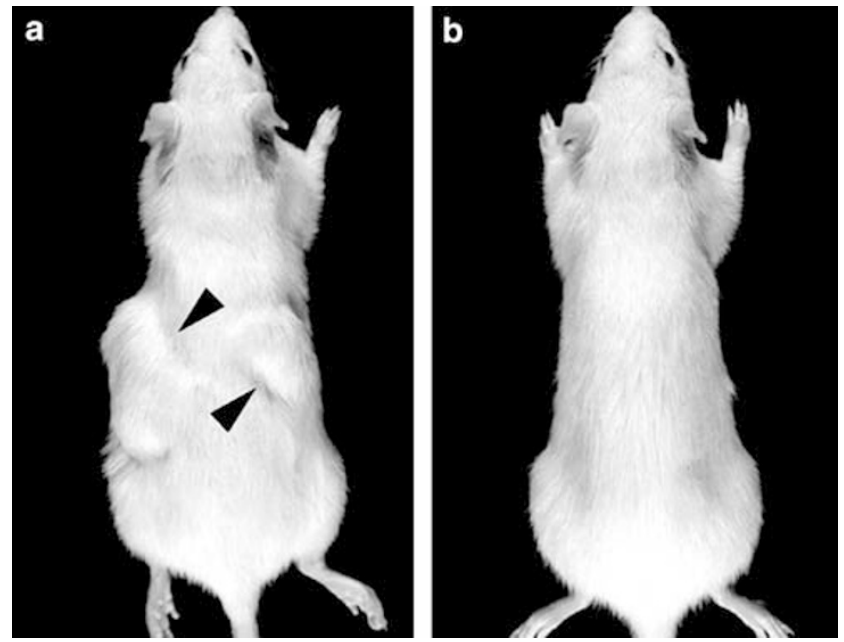

examined the genomic DNA of cloned cells from

Figure 2 Development of tumors at the subcutis in lck-pX rats inoculated with the transformed thymoma tissues. At 1 month after the inoculation, tumor formations were found when the transformed thymoma tissues (a, arrowheads) but not the originals (b) were injected.

malignant thymoma, the exon $1 \beta$ but not 2 or 3 was detected by PCR (Figure 6b). The gene similar to 5-MTAPase located in the downstream of the p16ink4a gene was detected, suggesting that the deletion of chromosome 5 included the exons $1 \alpha, 2$ and 3 of the p16ink $4 a$ gene in malignant thymomas.

\section{Discussion}

Although benign epithelial thymomas developed in lck-pX rats, no other disease or abnormality, such as malignancy of lymphocytes or immunological dysfunction etc, was observed because fatal dyspnea occurred due to expansive growth of the tumors in the thoracic cavity when young. ${ }^{10}$ To determine if thymomas can develop in a heterotopic situation in lck-pX rats, and acquire a long survival model carrying thymomas, we carried out heterotopic transplantation of lck-pX thymuses from newborns.
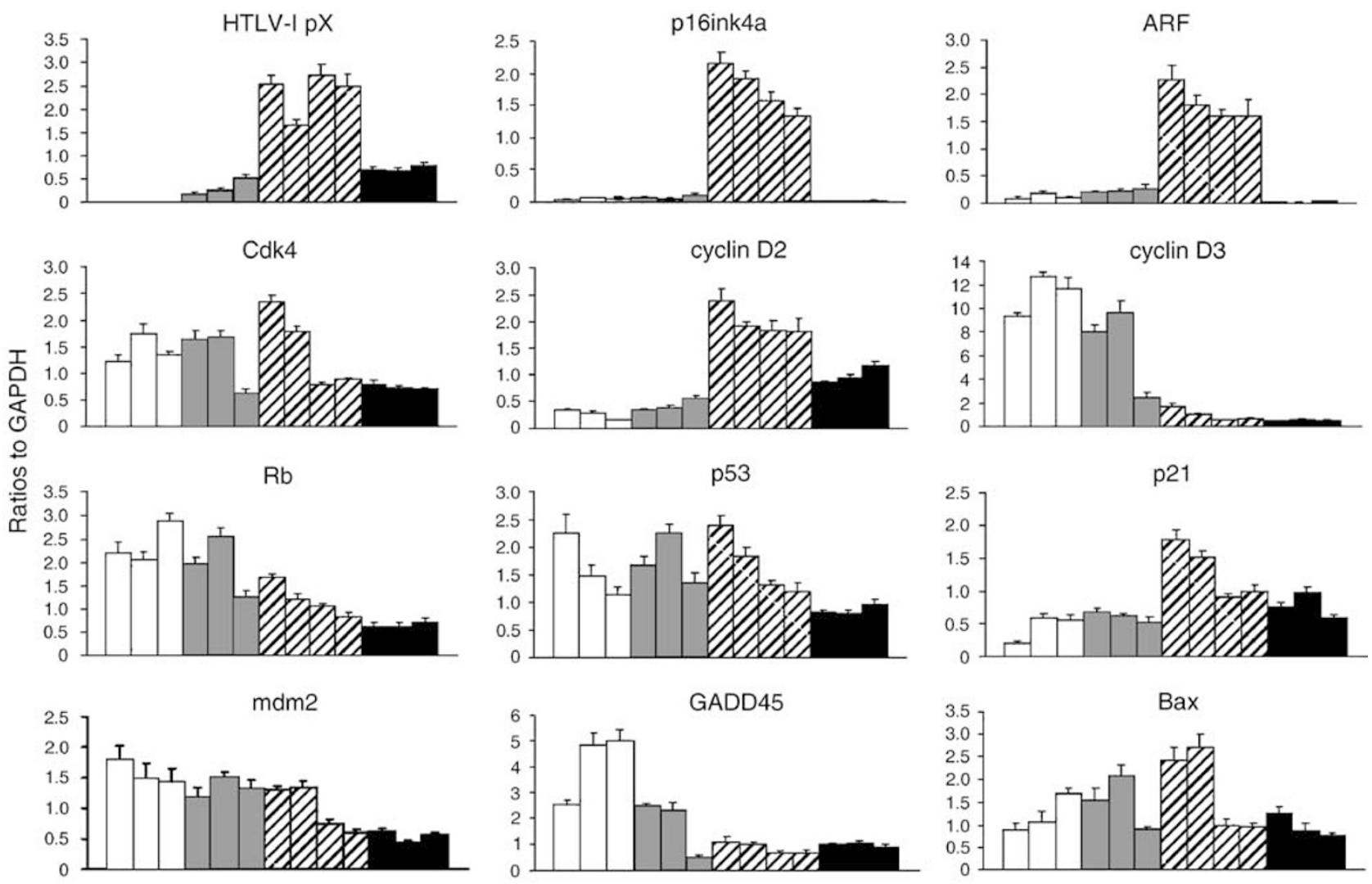

non-transgenic thymuses

Ick-pX thymuses without tumor

$\square$ original thymomas

malignant transformed thymomas

Figure 3 Comparisons of gene expressions among nonaffected thymuses and thymomas, using real-time quantitative RT-PCR. Total RNAs were isolated from nontransgnenic thymuses $(n=3)$, lck-pX thymuses without tumors $(n=3)$, original thymomas $(n=4)$ and thymomas with malignant transformation $(n=3)$, then real-time quantitative RT-PCR was performed. Prior to RNA isolation, thymocytes were disaggregated by rinsing the thymuses, the surface of which had been cut, in HBSS. It was histologically confirmed that over $90 \%$ of thymocytes were removed. Expression levels of $G A P D H$ were used for standardization of levels of each gene expression. Ratios to the expression levels of GAPDH in each sample are shown. Each column corresponds to an individual rat. Data are shown as mean \pm standard deviation on the basis of experiments performed in triplicate. 
a

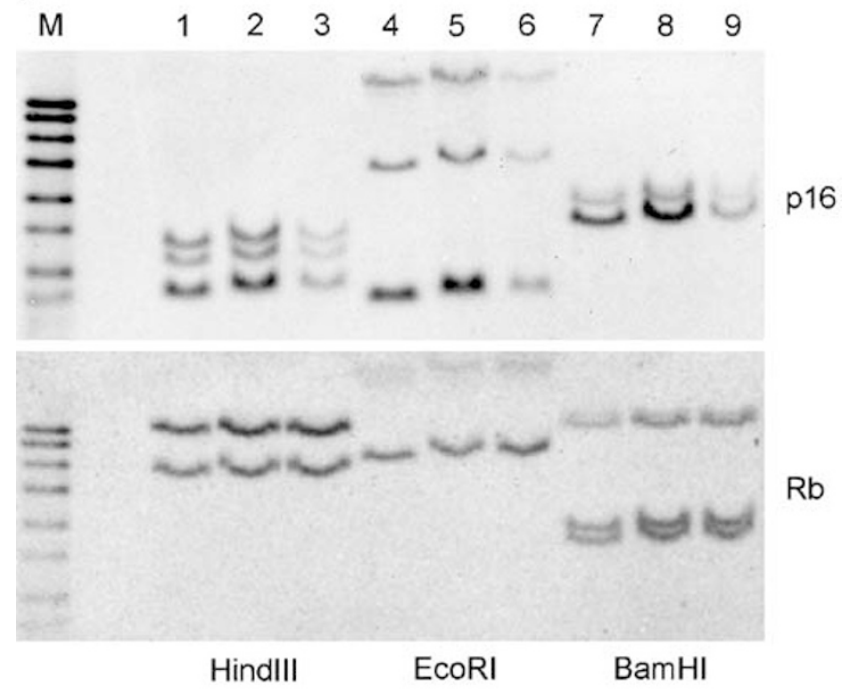

b

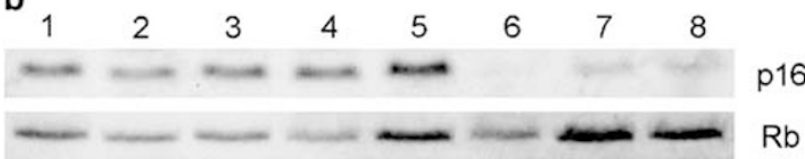

Figure 4 Genomic Southern blot analysis of the p16ink4a/ARF locus. (a) The upper panel: genomic DNAs from a nontransgenic thymus (lanes 1, 4, and 7), and original (lanes 2, 5, and 8) and transformed thymoma tissues (lanes 3,6, and 9) were digested with HindIII (lanes 1-3), BamHI (lanes 4-6) and EcoRI (lanes 7-9), then Southern hybridization was performed using the p16ink4a cDNA probe. Prior to RNA isolation, thymocytes were disaggregated by rinsing the thymuses, the surface of which had been cut, in HBSS. It was histologically confirmed that over $90 \%$ of thymocytes were removed. The lower panel: the $R b$ cDNA probe was used as a control for amounts of applied DNA samples. The representative results from repeated experiments are shown. $\mathrm{M}$ means a molecular size maker, DNA molecular weight marker VII, DIG-labeled (Roche Diagnostics). (b) The upper panel: comparison of densities of the representative band of the p16ink4a/ARF locus with HindIII digestion (corresponds with the lowest band in a, lanes 1-3) among the nontransgenic thymus (lane 1), original thymomas (lanes 2-5), and thymomas with malignant transformation (lanes 6-8). The lower panel: rehybridization using the $R b \mathrm{cDNA}$ probe for a control experiment. Each lane represents the genomic DNAs from tissues of different individuals.

The thymuses were transplanted into the subcapsular space of the kidney of other lck-pX rats that had been undergone thymectomy, where formation of new blood vessels is easily introduced into the grafted tissues. ${ }^{22}$ At 3 months after the transplantation, tumors with the same histological features as the original thymomas in lck-pX rats occupied the transplanted thymus in the recipient kidney, indicating that thymomas can also develop at heterotopic sites if there is a thymic tissue.

Interestingly, additional 3 months incubation could induce malignant transformation of thymomas. Similar malignant transformation was observed at a shorter period when the original thymomas were transplanted into the subcapsular space of the
1

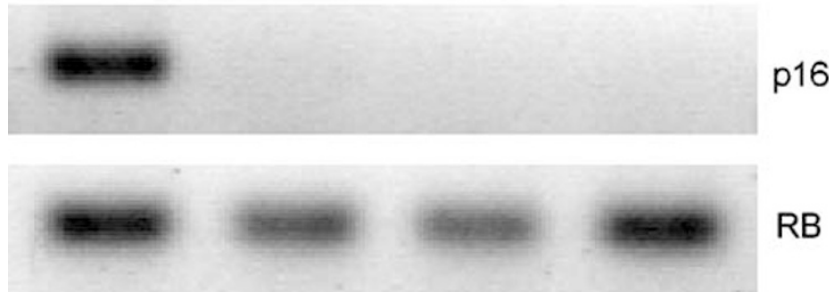

Figure 5 Detection of homozygous deletion of the p16ink4a exon $1 \alpha$ in cell clones from the thymoma with malignant transformation by genomic PCR. The upper panel: after establishment of cell clones from the transformed thymoma using the limiting dilution method, genomic PCR using the primer set specific for the p16ink $4 a$ exon $1 \alpha$ gene was performed used with each clone. The lower panel: amplifications of $R b$ gene served as a control for the DNA integrity. Lane 1 shows results of the normal rat genomic DNA as a control. Lanes 2-4 represent three different clones, respectively. Representative results from repeated experiments are shown.

kidney (unpublished data). These findings indicated that thymomas with a benign behavior developed in the transplanted thymuses, then malignant transformation occurred through an additional incubation period. Since we never found a malignant transformation of original thymomas $(n>100)$ in the thoracic cavity, ${ }^{10}$ environmental factors might be critical for the malignant transformation. Fidler ${ }^{23}$ noted that tumors could receive nutrients by diffusion when they were as small as $1-2 \mathrm{~mm}$ in diameter, but the angiogenesis was indispensable for bigger growth and to become aggressive and invasive. The subcapsular space of the kidney may be a suitable site for tumor aggression where vascularization into the grafted tissues is easily introduced. ${ }^{22}$ Actually, when tumor cells from the renal cell carcinoma were injected into subcutaneous, intraperitoneum, intravein or beneath the renal capsule of nude mice, a remarkable metastasis was observed only in cases where the cells were inoculated into the subcapsular space of the kidney. ${ }^{24}$ In line with this, the thymomas could develop and become malignant with aggressive growth in the subcapusular space of the kidney, although development of a tumor was never found when original thymomas were transplanted into the retroperitoneal subserosal space (unpublished data).

The most remarkable differences in host gene expressions related with p53 and $\mathrm{Rb}$ pathways between original and transformed thymomas were the expression of p16ink $4 a$ and $A R F$ genes. The p16ink4a and ARF are distinct tumor suppressor genes that share a common exon but encode nonhomologous proteins. ${ }^{25}$ Abnormalities of the p16ink4a/ARF locus were noted in $30-60 \%$ of human malignancies, such as esophageal carcinomas, gliomas, mesotheliomas and ATL. ${ }^{26,27}$ There was a report on the high expression of p16ink4a in HTLV-I-infected cell lines. ${ }^{28}$ The HTLV-I $p X$ gene product, Tax, which can trans-activate various cellular genes and is the most pathogenic factor of 


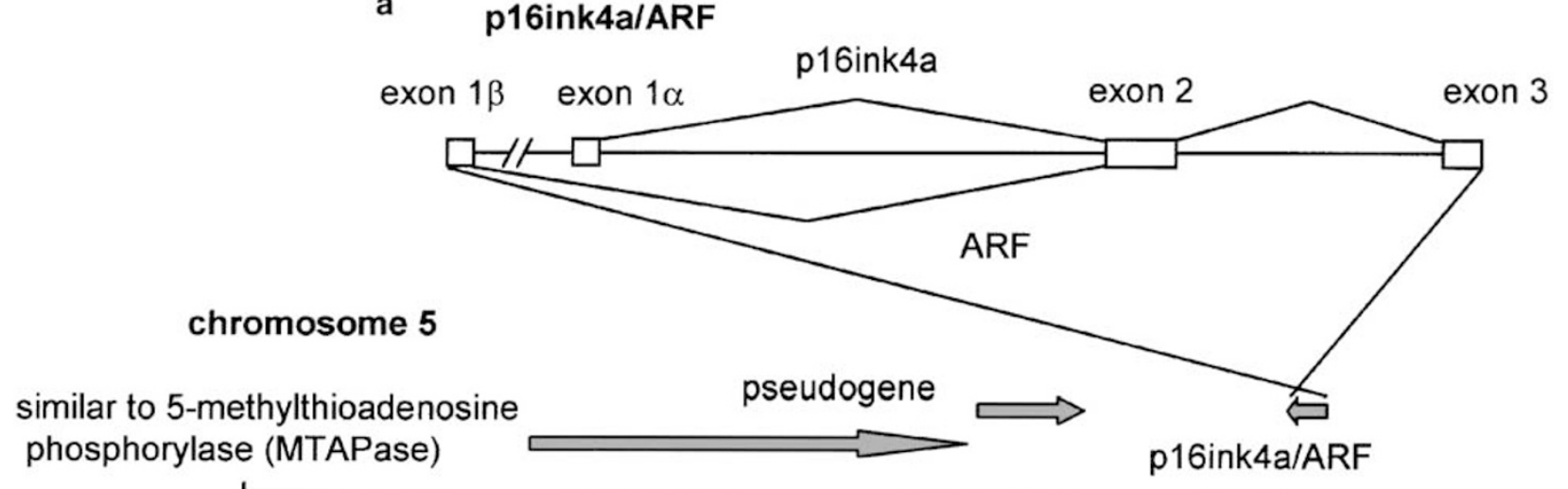

b

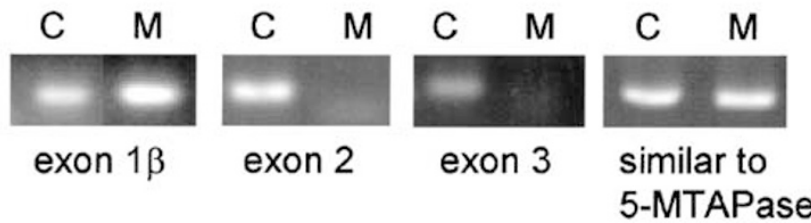

Figure 6 (a) Schematic representation of the loci of $p 16 i n k 4 a / A R F$ gene and its downstream on rat chromosome 5. The p16ink4a/ARF gene is mapped on the band $5 q 32$ in the reverse direction. The gene similar to 5-MTAPase is adjacent to the $p 16 i n k 4 a / A R F$ locus across a pseudogene. (b) Genomic PCR for the exons 1 $\beta, 2$, and 3, and the gene similar to 5-MTAPase. C: a control DNA sample derived from the rat synovial fibroblastic cell line, ${ }^{44}$ which is continuously maintained in our laboratory. M: the cloned cells from malignant thymoma. The representative results from repeated experiments are shown.

HTLV-I, ${ }^{11}$ increases the expression of cyclin D2 as an activator of the transcriptional factor, E2F, and suppresses the function of p16ink4a as an inhibitor of Cdk 4/6, which activate E2F together with cyclin D2 by protein-protein interaction, thus suggesting that the cell cycle may be deregulated in cells expressing Tax protein. ${ }^{14}$ In original thymomas, the high levels of p16ink4a expression may control the tumor growth with aberrant expressions of the $p X$ gene and cyclin $D 2$ gene trans-activated by Tax, and may suppress at least malignant cell transformation. Therefore, it seems likely that loss of the p16ink $4 a$ expression in the transformed thymoma cells induced a more prominent growth, although levels of the $p X$ expression in the malignant thymomas were less than a half of that in the originals. ARF, which is activated by $\mathrm{E} 2 \mathrm{~F}^{29,30}$ increases the functional potency of p53 by neutralization of mdm2 oncoprotein (a suppressor of p53) activity. ${ }^{31}$ Although the expression of $A R F$ was at high levels in the originals and was lost in the malignant thymomas, the $p 53$ gene may not be critical for the benign to malignant transformation in our model, because no significant difference in the expressions of $p 53$ and its target genes, such as GADD45, p21, mdm2, and bax, was seen between the original and transformed thymomas. Tolbert et al ${ }^{32}$ reported that ARF deficiency had no impact on functions of p53 and its related molecules in the transgenic brain tumor model. ARF-dependency of p53 and its related molecules may vary in cell types. However, some functions of p53 repressed by Tax ${ }^{12,13}$ might relate HTLV-I tumorigenesis in thymomas of lck-pX rats, because the expression levels of GADD45, one of the p53 target genes, were significantly suppressed not only in the transformed thymomas but also in the originals.

To clarify the loss of p16ink4a/ARF expression, chromosomal and genomic analyses were performed. Although several chromosomal abnormalities were evident in the transformed thymomas, no homozygous abnormality was found in chromosome 5, which encoded the p16ink4a/ARF locus in karyotype analysis. However, genomic Southern blots and PCR using the primer sets specific for the p16ink4a/ARF gene and its downstream gene similar to 5-MTAPase indicated homozygous deletion of the $p 16$ locus in the transformed thymomas. The deletion of chromosome 5 included the exons $1 \alpha, 2$, and 3 of the p16ink $4 a$ gene in malignant thymomas. These findings corresponded to the fact that the deletion was not recognized by the karyotype analysis. A similar deletion was found in some human squamous cell carcinomas of the head and neck. ${ }^{33}$ It seems likely that a certain site where the genome is liable to be lost in the p16ink $4 a$ gene, and this may be associated with malignant transformation of some kinds of tumors. Generally, inactivation of p16ink $4 a$ is caused by homozygous deletions, methylations of the promoter or point mutations, and the frequencies and patterns of p16ink4a inactivation are different depending on the origins of tumors. ${ }^{34}$ In cases of ATL, not only homozygous deletion of p16ink $4 a^{27,35}$ but also 
methylations of the p16ink4a promoter ${ }^{36}$ were demonstrated.

Expression levels of the $p X$ gene, the most pathogenic factor of HTLV-I, were significantly lower in the malignant thymomas than those in the originals, and were similar to tumor-free thymuses in lck-pX rats. Although the reason for this decrease of the $p X$ expression in malignant thymomas remains unclear, it was functionally confirmed by the decrease of cyclin D2 expression that is directly controlled by Tax through an NF- $\kappa \mathrm{B}$-like element and a CRE-like element residing in the promoter. ${ }^{37}$ Therefore, it was suggested that the $p X$ expression was not essential for growth of the thymomas after malignant transformation. In line with this, the defective proviruses, which could not express intact Tax, were frequently detected in the aggressive form of ATL, such as acute-type and lymphoma-type ATL. ${ }^{38,39}$ The collecting evidence suggests that the aggressive form of tumor cells may not require the expression of $p X$ gene for further growth, once they have reached to the state of a high malignancy with alteration of host genes for regulation of the cell cycle, such as p16ink4a/ARF. Moreover, loss of Tax on the tumor cells may lead to an escape from immune systems of the host, because Tax is a major target of cytotoxic $\mathrm{T}$ lymphocytes. ${ }^{40}$ Since Tax has mutagenic effects on cellular genes ${ }^{41}$ and the subcapsular space of the kidney provides a better environment, including the potential of vascularization and larger space limits than the thorax, for cell division of tumors with aberrant expression of Tax, accumulation of mutations of genes, including key molecules such as p16ink4a/ARF, may facilitate malignant transformation in thymomas with a benign behavior.

ATL can be classified into four clinical subtypes: chronic, smoldering, acute and lymphoma type..$^{42,43}$ The progression from indolent ATL (chronic and smoldering type) to aggressive ATL (acute and lymphoma type) is sometimes observed, and it is also known that abnormalities of p16ink4a and p53 frequently accompany this progression. ${ }^{27,35,43}$ Therefore, the malignant transformation seen in our animal model might correspond to the progression of ATL in humans. The in vivo model for malignant transformation described here appears useful for understanding the mechanism of malignant progression not only in ATL but also in other general benign tumors.

\section{Acknowledgements}

We thank the entire staff of the Institute of Animal Experimentation, Hokkaido University Graduate School of Medicine, for maintenance of transgenic rats, and Ken-ichi Nakase, Chisato Sudo and Masayo Tateyama for technical assistance. This work was supported by grants from the Ministry of Education, Culture, Sports, Science and Technology of Japan.

\section{References}

1 Poiesz BJ, Ruscetti FW, Gazdar AF, et al. Detection and isolation of type $C$ retrovirus particles from fresh and cultured lymphocytes of a patient with cutaneous T-cell lymphoma. Proc Natl Acad Sci USA 1980;77: 7415-7419.

2 Yoshida M, Miyoshi I, Hinuma Y. Isolation and characterization of retrovirus from cell lines of human adult T-cell leukemia and its implication in the disease. Proc Natl Acad Sci USA 1982;79:2031-2035.

3 Seiki M, Hattori S, Hirayama Y, et al. Human adult T-cell leukemia virus: complete nucleotide sequence of the provirus genome integrated in leukemia cell DNA. Proc Natl Acad Sci USA 1983;80:3618-3622.

4 Grassmann R, Dengler C, Muller-Fleckenstein I, et al. Transformation to continuous growth of primary human T lymphocytes by human T-cell leukemia virus type I X-region genes transduced by a Herpesvirus saimiri vector. Proc Natl Acad Sci USA 1989;86:33513355.

5 Tanaka A, Takahashi C, Yamaoka S, et al. Oncogenic transformation by the tax gene of human T-cell leukemia virus type I in vitro. Proc Natl Acad Sci USA 1990;87:1071-1075.

6 Hinrichs SH, Nerenberg M, Reynolds RK, et al. A transgenic mouse model for human neurofibromatosis. Science 1987;237:1340-1343.

7 Nerenberg M, Hinrichs SH, Reynolds RK, et al. The tat gene of human T-lymphotropic virus type 1 induces mesenchymal tumors in transgenic mice. Science 1987;237:1324-1329.

8 Grossman WJ, Kimata JT, Wong FH, et al. Development of leukemia in mice transgenic for the tax gene of human T-cell leukemia virus type I. Proc Natl Acad Sci USA 1995;92:1057-1061.

9 Yamada S, Ikeda H, Yamazaki $\mathrm{H}$, et al. Cytokineproducing mammary carcinomas in transgenic rats carrying the $\mathrm{pX}$ gene of human T-lymphotropic virus type I. Cancer Res 1995;55:2524-2527.

10 Kikuchi K, Ikeda $\mathrm{H}$, Tsuchikawa $\mathrm{T}$, et al. A novel animal model of thymic tumour: development of epithelial thymoma in transgenic rats carrying human $\mathrm{T}$ lymphocyte virus type I pX gene. Int J Exp Pathol 2002;83:247-255.

11 Yoshida M. HTLV-1 oncoprotein Tax deregulates transcription of cellular genes through multiple mechanisms. J Cancer Res Clin Oncol 1995;121:521-528.

12 Suzuki T, Uchida-Toita M, Yoshida M. Tax protein of HTLV-1 inhibits CBP/p300-mediated transcription by interfering with recruitment of $\mathrm{CBP} / \mathrm{p} 300$ onto DNA element of E-box or p53 binding site. Oncogene 1999; 18:4137-4143.

13 Pise-Masison CA, Radonovich M, Sakaguchi K, et al. Phosphorylation of p53: a novel pathway for p53 inactivation in human T-cell lymphotropic virus type 1-transformed cells. J Virol 1998;72:6348-6355.

14 Suzuki T, Kitao S, Matsushime H, et al. HTLV-1 Tax protein interacts with cyclin-dependent kinase inhibitor p16INK4A and counteracts its inhibitory activity towards CDK4. EMBO J 1996;15:1607-1614.

15 Jin DY, Spencer F, Jeang KT. Human T cell leukemia virus type 1 oncoprotein Tax targets the human mitotic checkpoint protein MAD1. Cell 1998;93:81-91.

16 Gatza ML, Watt JC, Marriott SJ. Cellular transformation by the HTLV-I Tax protein, a jack-of-all-trades. Oncogene 2003;22:5141-5149. 
17 Azran I, Schavinsky-Khrapunsky Y, Aboud M. Role of Tax protein in human T-cell leukemia virus type-I leukemogenicity. Retrovirology 2004;1:20.

18 Wittwer CT, Ririe KM, Andrew RV, et al. The LightCycler: a microvolume multisample fluorimeter with rapid temperature control. Biotechniques 1997;22: 176-181.

19 Wang HC, Fedoroff S. Banding in human chromosomes treated with trypsin. Nat New Biol 1972;235:52-54.

20 Sherr CJ. The Pezcoller lecture: cancer cell cycles revisited. Cancer Res 2000;60:3689-3695.

21 Tsuchikawa T, Ikeda H, Kikuchi K, et al. Hematopoietic progenitor cells as possible origins of epithelial thymoma in a human $\mathrm{T}$ lymphocyte virus type I pX gene transgenic rat model. Lab Invest 2004;84: 245-252.

22 Kumagai N, O’Neil JJ, Barth RN, et al. Vascularized islet-cell transplantation in miniature swine. I. Preparation of vascularized islet kidneys. Transplantation 2002;74:1223-1230.

23 Fidler IJ. Host-tumor interactions. Cancer Control 1998;5:8-9.

24 Naito S, von Eschenbach AC, Giavazzi R, et al. Growth and metastasis of tumor cells isolated from a human renal cell carcinoma implanted into different organs of nude mice. Cancer Res 1986;46:4109-4115.

25 Quelle DE, Zindy F, Ashmun RA, et al. Alternative reading frames of the INK4a tumor suppressor gene encode two unrelated proteins capable of inducing cell cycle arrest. Cell 1995;83:993-1000.

26 Sherr CJ. Cancer cell cycles. Science 1996;274:16721677.

27 Hatta Y, Hirama T, Miller CW, et al. Homozygous deletions of the p15 (MTS2) and p16 (CDKN2/MTS1) genes in adult T-cell leukemia. Blood 1995;85:26992704.

28 Akagi T, Ono H, Shimotohno K. Expression of cellcycle regulatory genes in HTLV-I infected T-cell lines: possible involvement of Tax1 in the altered expression of cyclin D2, p18Ink4 and p21Waf1/Cip1/Sdi1. Oncogene 1996;12:1645-1652.

29 Bates S, Phillips AC, Clark PA, et al. p14ARF links the tumour suppressors RB and p53. Nature 1998;395: 124-125.

30 Khleif SN, DeGregori J, Yee CL, et al. Inhibition of cyclin D-CDK4/CDK6 activity is associated with an E2F-mediated induction of cyclin kinase inhibitor activity. Proc Natl Acad Sci USA 1996;93:4350-4354.

31 Sherr CJ. Tumor surveillance via the ARF-p53 pathway. Genes Dev 1998;12:2984-2991.
32 Tolbert D, Lu X, Yin C, et al. p19 $9^{\mathrm{ARF}}$ is dispensable for oncogenic stress-induced p53-mediated apoptosis and tumor suppression in vivo. Mol Cell Biol 2002;22: 370-377.

33 Raschke S, Balz V, Efferth T, et al. Homozygous deletion of CDKN2A caused by alternative mechanisms in various human cancer cell lines. Gene Chromosomes Cancer 2005;42:58-67.

34 Rocco JW, Sidransky D. p16 (MTS-1/CDKN2/INK4a) in cancer progression. Exp Cell Res 2001;264:42-55.

35 Uchida T, Kinoshita T, Watanabe T, et al. The CDKN2 gene alterations in various types of adult T-cell leukaemia. Br J Haematol 1996;94:665-670.

36 Nosaka K, Maeda M, Tamiya S, et al. Increasing methylation of the CDKN2A gene is associated with the progression of adult T-cell leukemia. Cancer Res 2000;60:1043-1048.

37 Huang $\mathrm{Y}$, Ohtani K, Iwanaga $\mathrm{R}$, et al. Direct transactivation of the human cyclin D2 gene by the oncogene product Tax of human T-cell leukemia virus type I. Oncogene 2001;20:1094-1102.

38 Sakurai H, Kondo N, Ishiguro N, et al. Molecular analysis of a HTLV-I pX defective human adult T-cell leukemia. Leuk Res 1992;16:941-946.

39 Tamiya S, Matsuoka M, Etoh $\mathrm{K}$, et al. Two types of defective human T-lymphotropic virus type I provirus in adult T-cell leukemia. Blood 1996;88: 3065-3073.

40 Jacobson S, Shida H, McFarlin DE, et al. Circulating CD8+ cytotoxic T lymphocytes specific for HTLV-I pX in patients with HTLV-I associated neurological disease. Nature 1990;348:245-258.

41 Miyake H, Suzuki T, Hirai H, et al. Trans-activator Tax of human T-cell leukemia virus type 1 enhances mutation frequency of the cellular genome. Virology 1999;253:155-161.

42 Shimoyama M. Diagnostic criteria and classification of clinical subtypes of adult T-cell leukaemia-lymphoma. A report from the Lymphoma Study Group (1984-87). Br J Haematol 1991;79:428-437.

43 Sakashita A, Hattori T, Miller CW, et al. Mutations of the p53 gene in adult T-cell leukemia. Blood 1992; 79:477-480.

44 Abe A, Ishizu A, Ikeda $\mathrm{H}$, et al. Bone marrow cells carrying the env-pX transgene play a role in the severity but not prolongation of arthritis in human T-cell leukemia virus type-I transgenic rats: a possible role of articular tissues carrying the transgene in the prolongation of arthritis. Int J Exp Pathol 2004;85: 191-200. 